\title{
Antiproton production from cosmic-ray interactions and its compatibility with AMS-02 data
}

\author{
P. de la Torre Luque, ${ }^{a, *}$ D. Gaggero ${ }^{b}$ and M. N. Mazziotta ${ }^{c}$ \\ ${ }^{a}$ The Oskar Klein Centre, Department of Physics, Stockholm University, AlbaNova SE-10691 Stockholm, \\ Sweden \\ ${ }^{c}$ Istituto Nazionale di Fisica Nucleare, Sezione di Bari via Orabona 4, I-70126 Bari, Italy \\ ${ }^{b}$ Instituto de Física Teórica UAM-CSIC, Campus de Cantoblanco E-28049 Madrid, Spain \\ E-mail: pedro.delatorreluque@fysik.su.se
}

Recent cosmic-ray (CR) antiproton studies have claimed the possibility of an excess of data over the predicted flux at around $10 \mathrm{GeV}$, which can be the signature of dark matter annihilating into antiprotons. Nevertheless, this excess is subject to many uncertainties related to the evaluation of the antiproton spectrum produced from spallation interactions of CRs. In this work, we implement a combined MCMC analysis of the secondary ratios of B, Be and $\mathrm{Li}$ and the antiproton-to-proton spectrum $(\bar{p} / p)$, also including nuisance parameters to consider the uncertainties related to the spallation cross sections. This analysis allows us to constrain the Galactic halo height and the rest of propagation parameters, evaluate the impact of cross sections uncertainties in the determination of the antiproton spectrum and test the origin of the excess of antiprotons. We show that the $\bar{p} / p$ spectrum is compatible with a pure secondary origin within the uncertainties related. In particular, we find that the energy dependence of the evaluated $\bar{p} / p$ spectrum matches the AMS-02 data at energies above $3 \mathrm{GeV}$. In particular, flat residuals at the level of $10 \%$ are found with respect to the new AMS-02 $\bar{p}$ dataset, which is reasonably explained by a possible underestimation on the cross sections of antiproton production, which currently constitute the main source of uncertainties in the evaluation of their flux.

$3^{\text {th }}$ International Cosmic Ray Conference (ICRC 2021)

July 12 th - 23rd, 2021

Online - Berlin, Germany

\footnotetext{
${ }^{*}$ Presenter
} 


\section{Introduction}

Cosmic-ray particles constitute a precious tool for probing a variety of galactic processes. Current CR missions like AMS-02 [1-4], DAMPE [5], or Fermi [6], allow us test our standard models of the galactic environment and perform precise studies on their origin.

In particular, $\mathrm{CR}$ antinuclei represent an useful tool for revealing the existence of the elusive dark matter (DM), since it is theoretically expected that they (concretely WIMP particles) annihilate or decay into standard model particle-antiparticle pairs, or even study their emission from primordial black holes, provided that their production from CR interactions is relatively low. In fact, en the last years, many studies have shown difficulties to reproduce the antiprotons experimental data considering a purely secondary origin (i.e. produced from the interactions of CRs with the interstellar gas) and have claimed the necessity of adding an extra source of production, possibly coming from WIMPs. The clearest example is an apparent excess of antiprotons over the expected flux at 10-20 GeV, reported by different groups [7-11], which seems to fit very well with production from a WIMP particle of a mass around $50-100 \mathrm{GeV}$ and a thermally-averaged cross section near the thermal cross sections $\left(\langle\sigma v\rangle \sim 3 \times 10^{-26} \mathrm{~cm}^{3} / \mathrm{s}\right)$ annihilating to $b \bar{b}$ pairs. Nevertheless, the astrophysical uncertainties related to modelling the production of these antinuclei are still very high and have not been well taking into account until very recently (see, for instance $[12,13]$ ).

The total source term for secondary production of antiprotons from spallation reactions of CRs with the gas in the interstellar gas has form:

$$
Q_{C R+I S M \longrightarrow \bar{p}}\left(E_{\bar{p}}\right)=\sum_{C R} 4 \pi n_{I S M} \int_{0}^{\infty} d E \phi_{C R}(E) \frac{d \sigma_{C R+I S M \longrightarrow \bar{p}}}{d E_{\bar{p}}}\left(E, E_{\bar{p}}\right)
$$

where the key ingredients are the CR fluxes (the most abundant CR particles are protons and $\mathrm{He}$ ) and the $\bar{p}$ production cross sections. Notably, the determination of the cross sections of $\bar{p}$ production are subject to important uncertainties, and results in $12 \%$ to $20 \%$ uncertainties in the evaluation of the local $\bar{p}$ spectrum [14-16].

Further uncertainties in modelling the production of antiprotons are due to the evaluation of the propagation parameters, used to simulate the CRs diffusive motion and interactions in the Galaxy. The diffusion coefficient plays a crucial role here and its determination is performed by reproducing the $\mathrm{CR}$ secondary-to-primary flux ratios (mainly B/C). Unfortunately, the uncertainties related to the cross sections of production of secondary CRs are high $(>20 \%)$, so that they lead to high uncertainties in the determination of the diffusion coefficient too $[13,17,18]$. In addition, correlated errors in the AMS-02 data seem to lead to looser constraints in the determination of the propagation parameters, the solar modulation effect makes our prediction to be still uncertain at low energies and the parametrization of the diffusion coefficient below a few $\mathrm{GeV} / \mathrm{n}$ is not totally clear yet. In this work, a combination of different secondary species and their ratios is employed in order to reduce the impact of these uncertainties and study the predicted spectrum of antiprotons obtained assuming they are originated from CR collisions. 


\section{Analysis method}

Here, the propagation of CRs is numerically solved with the DRAGON2 code ${ }^{1}$ assuming a twodimensional model for the Galaxy structure, with cylindrical symmetry, as described in refs. [19] and [20]. This differential equation takes into account the diffusive motion of particles in the Galaxy (characterized by the spatial diffusion coefficient, $D(R)$ ), their reacceleration (characterized by the diffusion coefficient in momentum space, $D_{p p}$ ), convection, injection from sources, energy losses, decays and collisions with the interstellar medium (ISM). Also the height of the halo is an important parameter to consider in these computations.

The parametrization of the spatial diffusion coefficient employed in this work is a smoothly broken power-law, motivated by the break at high energies in the spectra of CRs, which is doubly pronounced in secondary CR particles [3]:

$$
D(R)=D_{0} \beta^{\eta} \frac{\left(R / R_{0}\right)^{\delta}}{\left[1+\left(R / R_{b}\right)^{\Delta \delta / s}\right]^{s}},
$$

where $R_{0}=4 \mathrm{GV}$ is the reference rigidity and the values of the rigidity break $\left(R_{b}\right)$, the change in spectral index ( $\Delta \delta=\delta-\delta_{h}$, where $\delta_{h}$ is the spectral index for $R>R_{b}$ ), and a smoothing parameter $s$ are: $\Delta \delta=0.14, R_{b}=312 \mathrm{GV}$ and $s=0.040$, as determined in Ref. [21]. The spatial diffusion coefficient and diffusion coefficient in momentum space are related by the Alfvén velocity, $V_{A}$, as $D_{p p} \times D \propto V_{A}^{2} p^{2}[22]$.

To simulate the effect of the solar modulation to the flux of CRs reaching Earth, we make use of the Force-Field approximation [23], characterized by the Fisk potential, $\phi$. Charge-sign modulation is applied here, following the approach developed in Ref. [24], which basically needs the introduction of another potential for the modulation of negatively charged particles, $\phi_{1}^{-}$. We follow a similar approach as described in Ref. [7] and obtain a value $\phi_{1}^{-}$around $0.9 \mathrm{GV}$. The standard Fisk potential is set to be in agreement to the data period of data collection from each dataset used here to compute our model. In particular, $\phi=0.58 \mathrm{GV}$ for the evaluation of the $\bar{p} / p$ spectrum [4].

In this work, we perform combined analyses of the secondary $\mathrm{CRs} \mathrm{B}, \mathrm{Be}$ and $\mathrm{Li}$, including also antiprotons $(\bar{p})$. The main propagation parameters $\left(\mathrm{H}, D_{0}, V_{A}, \eta\right.$ and $\left.\delta\right)$ are inferred from a fit to experimental data of the flux ratios of these CR species by means of the Markov-Chain Monte Carlo (MCMC) procedure presented in [18], and extended here to include the halo height value in the procedure. This analysis incorporates scale factors as nuisance parameters to adjust the normalization of the spallation cross sections of B, Be and Li production $\left(\mathcal{S}_{B}, \mathcal{S}_{B e}, \mathcal{S}_{L i}\right)$. The $\bar{p} / p$ spectrum, $\mathrm{B} / \mathrm{C}, \mathrm{B} / \mathrm{O}, \mathrm{Be} / \mathrm{C}, \mathrm{Be} / \mathrm{O}$ flux ratios (able to constrain the values of $D_{0} / \mathrm{H}, V_{A}, \eta$ and $\delta$ ), the $\mathrm{Be} / \mathrm{B}, \mathrm{Li} / \mathrm{B}, \mathrm{Li} / \mathrm{Be}$ ratios (which allow setting the values of the scaling parameters) and the ${ }^{10} \mathrm{Be} / \mathrm{Be}$ and ${ }^{10} \mathrm{Be} /{ }^{9} \mathrm{Be}$ flux ratios are included in this routine. The cross sections derived in Ref. [25] for the $\bar{p}$ production cross sections are used for the evaluation of the antiproton spectrum. Then, the production of antiprotons is scaled by a constant 5\% factor to take into account the contribution of heavier CR nuclei $(\mathrm{Z}>2)$ to their production in collisions with the ISM [14]. The experimental datasets used are the most recent ones from the AMS- 02 collaboration, except for the ${ }^{10} \mathrm{Be}$ ratios, which come from various experiments (see section 5 of Ref. [17]).

\footnotetext{
1actually, a well tested customized version: https://github.com/tospines/Customised-DRAGON2_beta
} 


\section{Results}

The propagation parameters obtained from this analysis are given in table 1, along with the statistical uncertainties of their determination. In table 2 we show the spectral index of the broken power-law used to parametrize the injection spectrum of primary CRs, with a break at $8 \mathrm{GeV} / \mathrm{n}$. The MCMC procedure is repeated after adjusting the injection parameters until the propagation parameters converge (i.e. the input propagation parameters are the same, within $1 \sigma$, as the output). As we see, the best fit halo height value, $\mathrm{H}$, is close to $6 \mathrm{kpc}$, compatible with recent analyses $[17,26]$. Interestingly, the value found for the effective Alfv/'en velocity, $V_{A}$, is compatible with no reacceleration, while typical analyses of secondary-to-primary rations predict a value close to $20-30 \mathrm{~km} / \mathrm{s}$ [18]. In addition, the value of the spectral index of the diffusion coefficient, $\delta$, is $\sim 0.49$, very close to the predicted value from a Kraichnan spectrum of turbulence in the interstellar medium, which has been also found in other recent analyses [27, 28].

The values obtained for the scale factors (used as nuisance parameters to scale the normalization of the DRAGON2 cross sections parametrization) are, $\mathcal{S}_{B}=0.96 \pm 0.01, \mathcal{S}_{B e}=0.89 \pm 0.01$ and $\mathcal{S}_{L i}=0.87 \pm 0.01$. As expected, the scale factor for the B production cross sections is the lowest, below $5 \%$. A renormalization factor of around $10 \%$ for the cross sections of Be and Li production is well reasonable too (see refs. [17, 18, 27, 28]) and, in fact, are similar to those obtained in Ref. [13] for the GALPROP cross sections parametrization.

In figure 1 we show some of the most important spectra of the ratios included in the analysis, as well as the spectra of proton and He, which are crucial to estimate the antiproton production. The $2 \sigma$ statistical uncertainties in the determination of the propagation parameters are also shown as bands in all panels. In the panel a), we report the flux ratios of $\mathrm{B}, \mathrm{Be}$ and $\mathrm{Li}$ to $\mathrm{C}$ (the ratios to $\mathrm{O}$ are in similar agreement). While a precise simultaneous fit to AMS-02 of all these ratios is obtained with the DRAGON2 cross sections, as shown in Ref. [18], the inclusion of the $\bar{p} / p$ spectrum in the analysis slightly worsens the fit of these ratios (although, the agreement is still at the level of $\sim 2 \sigma$ statistical uncertainty). We highlight that the use of AMS- 02 correlated errors would have significantly improved the fit to these ratios. Panel b) shows the ${ }^{10} \mathrm{Be} /{ }^{9} \mathrm{Be}$ spectrum, which is in

\begin{tabular}{|c|c|c|c|c|}
\hline \multicolumn{5}{|c|}{ Propagation parameters } \\
\hline$H(\mathbf{k p c})$ & $D_{0}\left(10^{28} \mathbf{c m}^{2} / \mathbf{s}\right)$ & $v_{A}(\mathbf{k m} / \mathbf{s})$ & $\eta$ & $\delta$ \\
\hline $6.07 \pm 0.11$ & $4.79 \pm 0.1$ & $0.28 \pm 1.25$ & $-1.57 \pm 0.08$ & $0.49 \pm 0.01$ \\
{$[5.82,6.27]$} & {$[4.59,5.01]$} & {$[0 ., 2.8]$} & {$[-1.75,-1.39]$} & {$[0.46,0.51]$} \\
\hline
\end{tabular}

Table 1: Summary of the results obtained in the combined analysis. The error given corresponds to the $1 \sigma$ uncertainty assuming the PDF to follow a Gaussian distribution. The actual $2 \sigma$ uncertainty range is given for every parameter within square brackets.

\begin{tabular}{|c|c|c|c|}
\hline \multicolumn{4}{|c|}{ Injection params ${ }^{1} \mathbf{H} \&{ }^{4} \mathbf{H e}$} \\
\hline${ }^{1} \mathbf{H} \gamma_{1}$ & ${ }^{1} \mathbf{H} \gamma_{2}$ & ${ }^{4} \mathbf{H e} \gamma_{1}$ & ${ }^{4} \mathbf{H e} \gamma_{2}$ \\
\hline 2.155 & 2.384 & 2.425 & 2.355 \\
\hline
\end{tabular}

Table 2: Injection parameters of $\mathrm{H}$ and He obtained for the fit to AMS-02 data. The MCMC procedure is repeated after adjusting the injection parameters until the propagation parameters converge. 
reasonable agreement with experimental data (although this is surrounded by huge uncertainties). Panel c) shows the secondary-to-secondary flux ratios of $\mathrm{B}, \mathrm{Be}$ and $\mathrm{Li}$, which are mainly dependent of the ratio between their production cross sections. A reasonably good agreement is obtained also here. Finally, the proton and He spectra are shown in panel d), which depend not only on the diffusion parameters, but also on the injection parameters (for more details, see Ref. [18]), tuned to reproduce these AMS-02 spectra (as well as $\mathrm{C}, \mathrm{O}, \mathrm{Ne}, \mathrm{Mg}$ and $\mathrm{Si}$ ). We remark that proton and He spectra are crucial in the evaluation of the $\bar{p}$ spectrum and the $\bar{p} / p$ ratio. In this panel, the uncertainties shown are both the $2 \sigma$ statistical uncertainties in the determination of the diffusion parameters and the solar modulation $(\sim \pm 0.12 \mathrm{GV})$. We include here solar modulation uncertainties in order to show that the treatment of solar modulation can also be important for $\mathrm{H}$ and $\mathrm{He}$ at low energies, since it leads to a different adjustment of their injection parameters.

a)

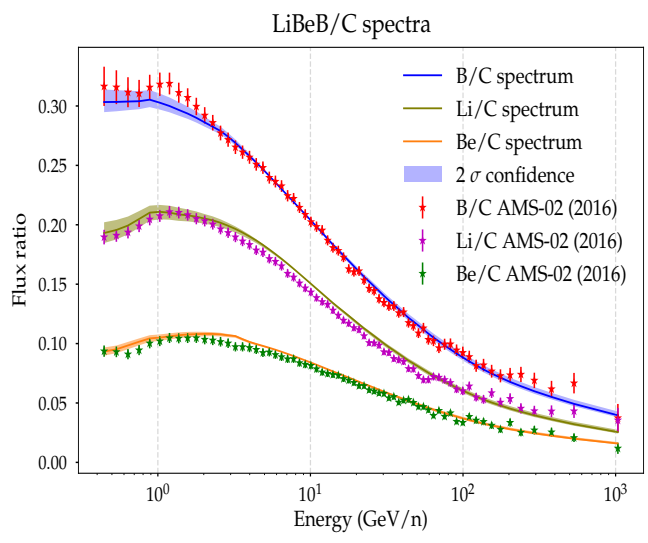

Sec/Sec spectra

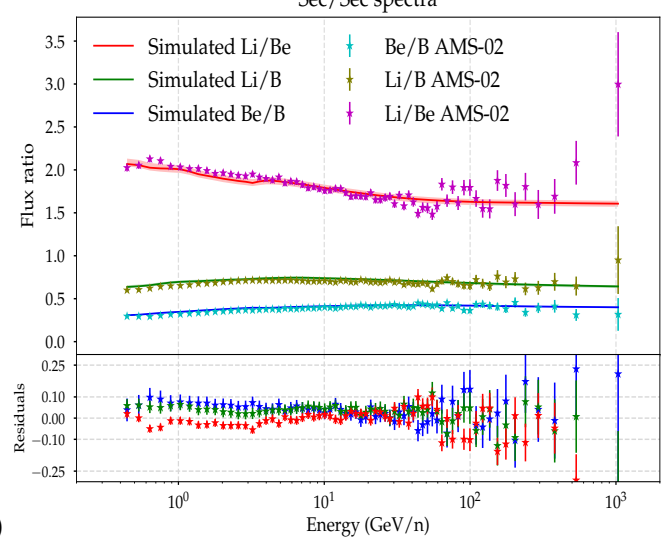

b)
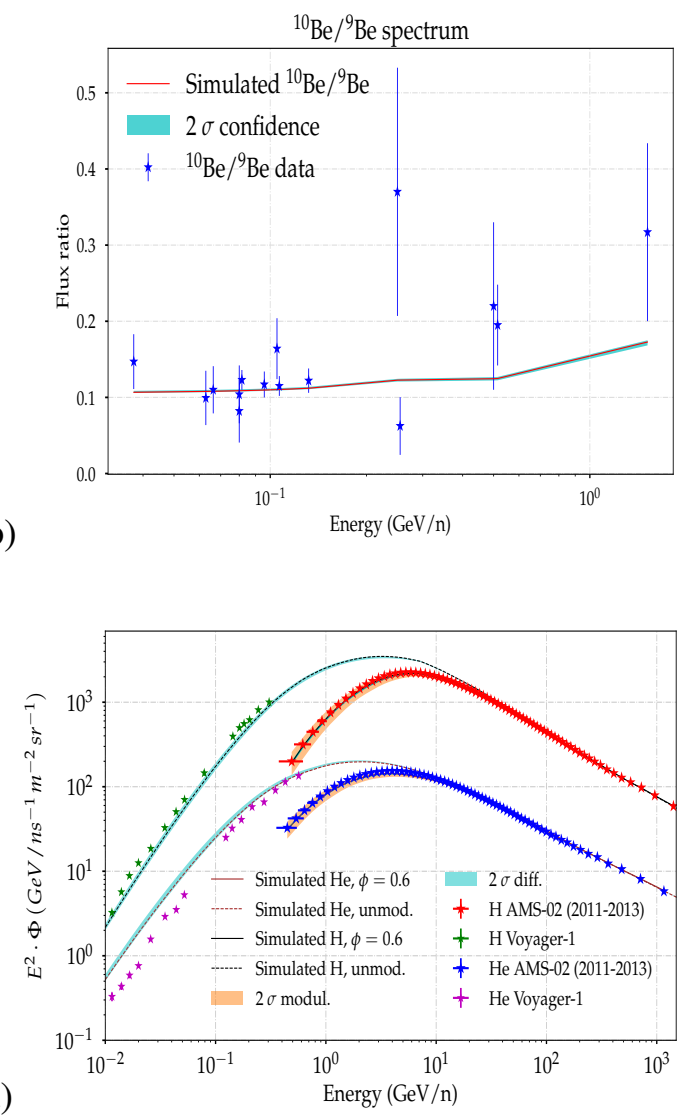

Figure 1: Relevant ratios involving secondary CRs produced in CR interactions with interstellar gas evaluated with the propagation parameters obtained in the combined analysis presented here and compared to experimental data. Panel a: flux ratios of $\mathrm{B}, \mathrm{Be}$ and $\mathrm{Li}$ to $\mathrm{C}$. Panel b: ${ }^{10} \mathrm{Be} /{ }^{9} \mathrm{Be}$ flux ratio. Panel c: secondary-to-secondary flux ratios of $\mathrm{B}, \mathrm{Be}$ and $\mathrm{Li}$ and the residuals with respect to AMS-02 data, defined as (model-data)/data. Panel d: Proton and He spectra compared to AMS-02 data (modulated spectra) and Voyager-1 (unmodulated spectra). Error bands represent the $2 \sigma$ statistical uncertainty in the determination of the propagation parameters and panel $\mathrm{d}$ also those from related to solar modulation (uncertainties in the determination of the Fisk potential). 


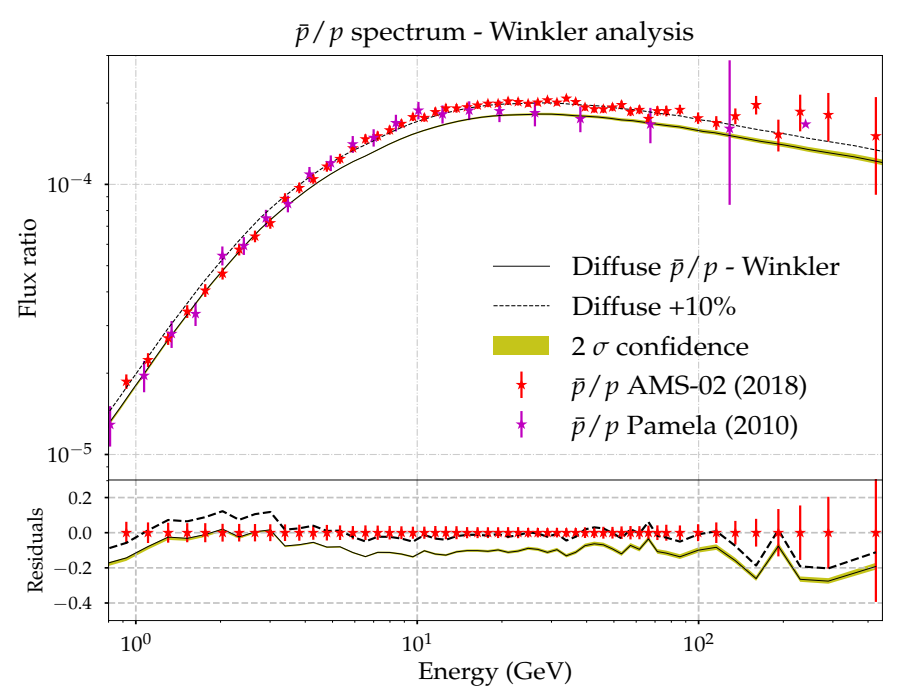

Figure 2: $\bar{p} / p$ spectrum evaluated with the propagation parameters determined in the combined $\bar{p} / p$ analysis. The statistical uncertainty in the evaluation of the model is shown as a yellow band and residuals with respect to AMS-02 data (2018) are also shown. The error bars on AMS-02 data are the $1 \sigma$ errors reported by the collaboration. On top of this, the same spectrum but scaled by a $10 \%$ is also shown as a dashed line, allowing us to see that a simple $10 \%$ scaling would make us reproduce experimental data within $1 \sigma$ errors.

The obtained $\bar{p} / p$ spectrum predicted from these propagation parameters (black solid line) is shown in figure 2. We emphasize here the importance of using the last release of antiprotons data by AMS-02 and the fact that we do not include possible correlations in the AMS-02 datasets. As it can be seen from the flat residuals (defined here as model-data/data, where the data are those from AMS-02) shown in this figure, the energy dependence predicted by our model is in very good agreement to the AMS- 02 data above $\sim 3 \mathrm{GeV}$, without any need to invoke extra sources producing antiprotons. In fact, this discrepancy does not resemble the typical bump-like structure that one would expect from possible extra sources of antiprotons, as from evaporation of primordial black holes or from a WIMP particle annihilating or decaying into hadronic final states. These residuals are at the level of $10 \%$ in the energy range above $\sim 3 \mathrm{GeV} / \mathrm{n}$. Therefore, the same prediction but scaled by $10 \%$ is shown as a dashed line. This offset can be very plausibly explained by a the uncertainties related to the cross sections of antiproton production, which are estimated to be of the level of $\sim 20 \%$ [14].

Finally, we highlight that the scale factors introduced to renormalize the cross sections of $\mathrm{B}, \mathrm{Be}$ and Li production play a major role in this analysis, allowing us to readjust the diffusion parameters (mostly determined by the ratios $\mathrm{B} / \mathrm{C}, \mathrm{B} / \mathrm{O}, \mathrm{Be} / \mathrm{C}$ and $\mathrm{Be} / \mathrm{O}$ ) to improve the predicted $\bar{p} / p$ spectrum with respect to data, while keeping a good agreement to the other secondary ratios. 


\section{Conclusions}

In this work, we have studied the diffuse $\bar{p} / p$ spectrum produced in CR interactions with the interstellar gas. We employ a combined analysis in which we have into account different secondary CRs and their ratios, as well as nuisance parameters that allow us to renormalize the cross sections parametrizations of production of $\mathrm{B}, \mathrm{Be}$ and $\mathrm{Li}$. These observables allow us to determine the main propagation parameters $\left(\mathrm{H}, D_{0}, V_{A}, \eta\right.$ and $\delta$ ) able to provide a simultaneous fit of the ratios of the different CR species. The analysis leads to a prediction of the $\bar{p} / p$ spectrum whose energy dependence is in very good agreement with the AMS-02 experimental data, recently released [4], above $3 \mathrm{GeV}$ and at the level of $\sim 10 \%$. In addition, the discrepancy found does not resemble the typical signal expected from dark matter annihilation, so that we argue that this discrepancy seems to be more plausibly explained by just a $10 \%$ scaling of the cross sections of $\bar{p}$ production. The use of the new $\bar{p} / p$ AMS-02 data has an important impact in the conclusions reached, since the residuals found are much flatter than when the old AMS-02 dataset is employed.

Significantly, we show that the energy dependence of the $\bar{p} / p$ spectrum is well reproduced assuming a pure secondary origin of antiprotons and that the excess found is plausibly explained by a rescaling of the cross sections of $\bar{p}$ production. Therefore, we conclude that taking into account all the sources of uncertainties in the evaluation of the secondary antiprotons produced from $\mathrm{CR}$ interactions with the ISM gas allows as to explain the $\bar{p} / p$ spectrum without any need of an extra source of production of $\bar{p}$. On top of this, we show that all these CR observables can be explained by the simple phenomenological prescription exposed above, at least within $2 \sigma$.

The direct inputs and outputs from the DRAGON2 runs with the propagation parameters obtained from this analysis are available at this repository ${ }^{2}$, as well as the best-fit spectra of proton, helium and antiproton.

2https://github.com/tospines/Analyses-and-plotting-codes/tree/main/DRAGON2_Antiprotons 


\section{References}

[1] M. Aguilar et al. (AMS), Phys. Rev. Lett. 114, 171103 (2015).

[2] M. Aguilar et al. (AMS Collaboration), Phys. Rev. Lett. 119, 251101 (2017).

[3] M. Aguilar et al. (AMS Collaboration), Phys. Rev. Lett. 120, 021101 (2018).

[4] M. Aguilar et al., Physics Reports 894, 1 (2021).

[5] Q. An et al., Science Advances 5 (2019), 10.1126/sciadv.aax3793.

[6] M. Ackermann et al., The Astrophysical Journal 750, 3 (2012).

[7] A. Reinert and M. W. Winkler, JCAP 01, 055 (2018), arXiv:1712.00002 [astro-ph.HE] .

[8] M.-Y. Cui et al., JCAP 06, 024 (2018), arXiv:1803.02163 [astro-ph.HE] .

[9] A. Cuoco, M. Krämer, and M. Korsmeier, Phys. Rev. Lett. 118, 191102 (2017).

[10] J. Heisig, Mod. Phys. Lett. A 36, 2130003 (2021), arXiv:2012.03956 [astro-ph.HE] .

[11] I. Cholis, T. Linden, and D. Hooper, Phys. Rev. D 99, 103026 (2019).

[12] M. Boudaud et al., Phys. Rev. Research 2, 023022 (2020).

[13] P. De la Torre Luque et al., J. Phys. Conf. Ser. 1690, 012010 (2020).

[14] M. Korsmeier, F. Donato, and M. Di Mauro, Phys. Rev. D 97, 103019 (2018).

[15] A. Cuoco et al., Phys. Rev. D 99, 103014 (2019).

[16] M. di Mauro, F. Donato, A. Goudelis, and P. D. Serpico, Phys. Rev. D 90, 085017 (2014).

[17] P. de la Torre Luque et al., JCAP 03, 099 (2021), arXiv:2101.01547 [astro-ph.HE] .

[18] P. De La Torre Luque et al., Accepted in JCAP (2021), arXiv:2102.13238 [astro-ph.HE] .

[19] C. Evoli et al., JCAP 02, 015 (2017), arXiv:1607.07886 [astro-ph.HE] .

[20] C. Evoli et al., JCAP 07, 006 (2018), arXiv:1711.09616 [astro-ph.HE] .

[21] Y. Génolini et al., Phys. Rev. Lett. 119, 241101 (2017), arXiv:1706.09812 [astro-ph.HE] .

[22] E.-S. Seo and V. S. Ptuskin, ApJ 431, 705 (1994).

[23] L. J. Gleeson and I. H. Urch, Astrophysics and Space Science 25, 387 (1973).

[24] I. Cholis, D. Hooper, and T. Linden, Phys. Rev. D 93, 043016 (2016), arXiv:1511.01507 .

[25] M. W. Winkler, JCAP 02, 048 (2017), arXiv:1701.04866 [hep-ph] .

[26] C. Evoli et al., Phys. Rev. D 101, 023013 (2020), arXiv:1910.04113 [astro-ph.HE] .

[27] N. Weinrich et al., Astron. Astrophys. 639, A131 (2020), arXiv:2002.11406 [astro-ph.HE] .

[28] M. Korsmeier and A. Cuoco, Physical Review D 103 (2021), 10.1103/physrevd.103.103016. 